\title{
Longitudinal microcomputed tomography-derived biomarkers for lung metastasis detection in a syngeneic mouse model: added value to bioluminescence imaging
}

\author{
Eyra Marien', Amy Hillen², Frank Vanderhoydonc ${ }^{1}$, Johannes V Swinnen ${ }^{1}$ and Greetje Vande Velde ${ }^{2}$
}

With more patients dying from metastasis than from primary cancers, metastasis is a very important area in cancer research. Investigators thereby heavily rely on animal models of metastasis to common organs such as the lung to improve our insight into the pathogenesis and to research novel therapeutic approaches to combat metastasis. In this experimental context, novel tools that allow longitudinal monitoring of lung metastasis in individual animals are highly needed. We have therefore evaluated for the first time microcomputed tomography $(\mu \mathrm{CT})$ as a very efficient and crossvalidated means to noninvasively and repeatedly monitor metastasis to the lung in individual, free-breathing syngeneic mice. Two individual clones of KLN205 cancer cells were intravenously injected in syngeneic DBA/2 mice and lung metastasis was monitored weekly during 3 weeks using $\mu \mathrm{CT}$, and was compared with the current gold standard histology and bioluminescence imaging (BLI). $\mu \mathrm{CT}$ enabled us to visualize diffuse tumor morphology and also to extract four different biomarkers that quantify not only tumor load but also aerated space in the lung as a marker of vital lung capacity and potential compensatory mechanisms. Complementary to $\mathrm{BLI}$, applying this novel $\mu \mathrm{CT}$-based approach enabled us to unravel sensitively and efficiently differences in metastatic potential between two cellular clones. In conclusion, $\mu \mathrm{CT}$ and $\mathrm{BLI}$ offer biomarkers that describe different and complementary aspects of lung metastasis, underlining the importance of multimodality follow-up. The added value of $\mu \mathrm{CT}$ findings is important to better assess lung metastasis and host/lung response in preclinical studies, which will be valuable for translational applications.

Laboratory Investigation (2017) 97, 24-33; doi:10.1038/labinvest.2016.114; published online 21 November 2016

Cancer is a group of disease characterized by the uncontrolled growth and spread of abnormal cells and is a leading cause of death worldwide. Today, cancer accounts globally for one in every eight deaths. In 2012, there were an estimated 14.1 million cases of cancer diagnosed and 8.2 million deaths from the disease around the world. Moreover, the global cancer burden is growing at an alarming pace: in 2030 alone, $~ 21.7$ million new cancer cases and 13.0 million cancer deaths are expected to occur, simply due to the growth and aging of the population. ${ }^{1}$

With more patients dying from metastasis than from primary cancers, metastasis is a very important area of ongoing research. ${ }^{2,3}$ Investigators thereby heavily rely on animal models of metastasis to common organs to improve our insight into the pathogenesis and to research novel therapeutic approaches to combat metastasis. One such important preclinical mouse model to measure and quantify metastasis in vivo is based on injection of tumor cells directly to the systemic circulation via tail vein injection, which results primarily in pulmonary metastasis, ${ }^{4,5}$ independently of the tumor type from which the injected tumor cells originate. In previous studies, mostly human-mouse xenograft models were used to study lung metastasis, in which human cancer cell lines are injected into immunocompromised mice. Disadvantages of these models include that tumors are a mosaic of human cancer cells and murine stromal cells, which can influence metastasis. Moreover, as the murine host needs to be immunocompromised in order for human tumors to grow, the ability to examine the role of the immune system in metastasis progression is lost in these models. In addition, specific features of immunocompromised mice may influence the biology and therefore the study of metastasis. ${ }^{4-6}$

\footnotetext{
'Laboratory of Lipid Metabolism and Cancer, Department of Oncology, LKI—Leuven Cancer Institute, KU Leuven—University of Leuven, Leuven, Belgium and ²Biomedica MRI/ MoSAIC, Department of Imaging and Pathology, KU Leuven_University of Leuven, Leuven, Belgium

Correspondence: Dr G Vande Velde, MScEng, PhD, Biomedical MRI/MoSAIC, Department of Imaging and Pathology, KU Leuven—University of Leuven, Gasthuisberg, O\&N I Herestraat 49-Box 505, Leuven 3000, Belgium.

E-mail: greetje.vandevelde@kuleuven.be

Received 17 March 2016; revised 30 September 2016; accepted 30 September 2016
} 
Moreover, because immunotherapy - in which the patient's immune system is used to treat cancer- is a highly emerging field in cancer therapy, ${ }^{7,8}$ syngeneic mouse models that have a functional immune system are gaining interest in cancer research.

Currently, lung metastasis is mostly detected and measured via gold standard histopathological analysis and other ex vivo readouts. Those analyses enable to study different molecular and cellular factors. However, an important disadvantage of ex vivo readouts is that they only offer data from one time point per animal, whereas the process of metastasis is in essence dynamic in space and time and highly variable. This warrants the need for novel research tools that allow longitudinal measurements in individual animals. In the past 20 years, in vivo bioluminescence imaging (BLI) has gained potential for longitudinal measurement of lung metastasis and is more and more used in preclinical studies. Research groups have indeed described a correlation between BLI signal intensity and tumor mass. ${ }^{9,10}$ Other advantages of BLI include its sensitivity as well as its cost efficiency and ease of use. Nevertheless, BLI suffers from several limitations, including poor spatial resolution, its restriction to preclinical applications because of the requirement for genetically encoded luciferase, and the dependence of light signal on tissue depth. ${ }^{11}$

In this context, in vivo microcomputed tomography $(\mu \mathrm{CT})$ imaging appears to be an extremely interesting imaging alternative to monitor lung metastasis as it captures longitudinal information and delivers visual and quantitative information about the whole lung in three dimensions with high resolution and sensitivity. Moreover, it allows the extraction of four different quantitative lung biomarkers (lung tissue volume, aerated lung volume, total lung volume, and mean lung density) from repetitive in vivo $\mu \mathrm{CT}$ scans of free-breathing animals. ${ }^{12}$ Our aim was to evaluate the behavior of mentioned $\mu \mathrm{CT}$-derived biomarkers for the first time in a preclinical syngeneic model of lung metastasis and whether they provide complementary information in addition to BLI. To further evaluate both imaging modalities, we have studied their correlation and tested whether they can differentiate the metastatic potential of different cellular clones.

\section{MATERIALS AND METHODS \\ Cell Culture}

The squamous cell carcinoma (SCC) lung cancer cell line KLN205 was obtained from the European Collection of Cell Cultures (Public Health England, Salisbury, UK; January 2014) and cultured in RPMI1640 medium (Life Technologies, Carlsbad, CA, USA) supplemented with $10 \%$ fetal bovine serum (Life Technologies), L-glutamine ( $2 \mathrm{mM}$; Life Technologies) and penicillin/streptomycin (Life Technologies). Cells were kept at $37^{\circ} \mathrm{C}$ in a humidified atmosphere of $5 \% \mathrm{CO}_{2}$ and regularly tested for mycoplasma contamination (VenorGEM Kit; Minerva Biolabs, Berlin, Germany). To prepare single-cell clones, single cells were grown as individual cell lines. Nine individual cell lines were grown, of which two were selected, based on similar cell morphology and in vitro growth characteristics compared with the parental cell line. Those two cell lines (clone 1 and clone 2) and the parental cell line were subsequently stably transduced with lentiviral vectors carrying a 3flag-fLuc-T2A-eGFP-Ires-Bsd construct, to introduce luciferase followed by selection using blasticidin $(6 \mu \mathrm{g} / \mathrm{ml}$; Life Technologies), without further single-cell cloning and expansion.

\section{Cell Proliferation Assay}

Cells were seeded in a 96-well plate (1000 cells per well) and proliferation curves were obtained with an IncuCyte Zoom system (Essen BioScience, Ann Arbor, MI, USA), which determined the percentage of confluence based on phase contrast images taken at $2 \mathrm{~h}$-intervals for the duration of the experiments.

\section{Animal Model}

Cells $\left(4 \times 10^{5}\right.$ cells in $200 \mu \mathrm{l}$ PBS) were injected in 8-week-old female DBA/2 mice (Envigo, Venray, The Netherlands) via tail vein injection. Eight animals were injected with cell clone 1 and nine animals with cell clone 2. Metastasis to the lungs was monitored using BLI and $\mu \mathrm{CT}$ at baseline and once a week during 3 weeks after injection. Mice were killed after 3 weeks and lungs were collected for histopathology, and fixed in $10 \%$ formalin in PBS for $24 \mathrm{~h}$. After paraffin embedding, sections were cut and stained with hematoxylin-eosin.

To study the effect of repeated $\mu \mathrm{CT}$ scanning, cells $\left(10^{5}\right.$ cells in $200 \mu \mathrm{l} \mathrm{PBS}$ ) were injected in 8-week-old female DBA/2 mice (Envigo). One group of mice $(n=6)$ was weekly scanned via $\mu \mathrm{CT}$, whereas a second control group $(n=6)$ was not. Four weeks after injection, lung metastasis was monitored using BLI and $\mu \mathrm{CT}$ for both control and scanned mice.

All animal experiments were carried out in compliance with National and European regulations. Ethical approval was obtained from the ethical committee at the KU Leuven. The mice were kept in individually ventilated cages with free access to food and water.

\section{Microcomputed Tomography}

Retrospectively respiratory-gated $\mu \mathrm{CT}$ data sets of freebreathing, anesthetized mice (1.5-2\% isoflurane in $100 \%$ oxygen) were acquired on a dedicated small animal $\mu \mathrm{CT}$ scanner (SkyScan 1076, software version 4.2; Bruker microCT, Kontich, Belgium). ${ }^{13}$ Briefly, images were acquired in list mode with the following parameters: $50 \mathrm{kVp}$ X-ray source voltage and $180 \mu \mathrm{A}$ current combined with a composite X-ray filter of $0.5 \mathrm{~mm}$ aluminum, $120 \mathrm{~ms}$ exposure time per projection, 9 projections per view, acquiring projections with $0.7^{\circ}$ increments over a total angle of $180^{\circ}$, and $2 \mathrm{~cm}$ field of view covering the lungs, producing four reconstructed $3 \mathrm{D}$ data sets with $35 \mu \mathrm{m}$ isotropic reconstructed voxel size corresponding to four different phases of the 
breathing cycle (4D). The number of phases was optimally set for our image acquisition settings, resulting in averaging of at least two projection images per breathing phase. Here we report data at the end of expiration. Total scanning time per mouse was $\sim 12 \mathrm{~min}$, resulting in a radiation dose of 813 mGy. ${ }^{14}$

Software provided by the manufacturer (TSort, NRecon, DataViewer, and CTan) was used to retrospectively gate, reconstruct, visualize, and process $\mu \mathrm{CT}$ data as described. ${ }^{15}$ Quantification of the mean lung density (CT number, expressed in Hounsfield Unit (HU) with the main grayscale index of water (81.32) set at $0 \mathrm{HU}$ and grayscale index of air (0) at $-1000 \mathrm{HU}),{ }^{13}$ lung tissue (including tumor) volume, aerated lung volume, and total lung volume was carried out for a volume-of-interest covering the lung, comprising of regions of interest that were manually delineated on the coronal $\mu \mathrm{CT}$ images, thereby avoiding the heart and main blood vessels. The threshold used to distinguish aerated from lung tissue volume was manually set at $-385 \mathrm{HU}$ and kept constant for all data sets.

\section{Bioluminescence Imaging}

In vitro BLI and BLI of mice under isoflurane gas anesthesia were carried out on an IVIS Spectrum System (CaliperLS; Perkin-Elmer, Hopkinton, MA, USA). Living Image software (version 4.4.17504) was used for quantifying luminescence.

To measure the in vitro BLI signal intensity, both control and transduced cells were equally seeded (60 000 cells per well in a 24-well plate) and measured the next day after $5 \mathrm{~min}$ incubation at $37^{\circ} \mathrm{C}$ with D-luciferin (Promega BeNeLux, Leiden, The Netherlands; $150 \mu \mathrm{g} / \mathrm{l}$ dissolved in PBS).

For in vivo BLI measurements, D-luciferin $(126 \mathrm{mg} / \mathrm{kg}$ dissolved in PBS $(15 \mathrm{mg} / \mathrm{ml})$ ) was injected intraperitoneally and acquisition of consecutive frames was started until maximum signal intensity was reached. Image acquisition numbers and times varied between 10 and 15 frames of
30-60 s each, depending on the optimal acquisition settings in function of the signal intensity intrinsic to the grade of lung metastasis. We measured the photon flux per second $(\mathrm{p} / \mathrm{s})$ through a region of interest $(2.9 \mathrm{~cm} \times 1.8 \mathrm{~cm})$ covering the lungs.

\section{Statistics}

Statistical tests were carried out in GraphPad Prism (version 6.0c; GraphPad Prism Software, San Diego, CA, USA). Twoway ANOVA with repeated measurements and matching Sidak's multiple comparisons tests were used to analyze the differences between different time points compared with baseline values and between different cell clones. Whenever a data point for an animal was missing due to premature killing, the longitudinal data for this animal was not included in the analysis. Spearman's correlations were used to evaluate the agreement between quantitative $\mu \mathrm{CT}$ and BLI parameters. ${ }^{16} \quad{ }^{\star} P<0.05, \quad{ }^{* *} P<0.01, \quad{ }^{* *} P<0.001$, ${ }_{* * \star *} P<0.0001$, and NS, not significant.

\section{RESULTS}

\section{Onset and Progression of Lung Metastasis can be Monitored via BLI}

To study lung metastasis in a preclinical mouse model, the cancer cell line KLN205 was intravenously injected in syngeneic DBA/2 mice. KLN205 cells are known to form metastatic lesions in lungs after inoculation into syngeneic mice. ${ }^{17,18}$ To assess the ability of the explored imaging methods to discriminate possible differences in metastatic capacity of tumor cells, two individual KLN205 lung cancer cell clones were prepared and engineered to stably express luciferase. To evaluate lung metastasis via BLI, mice were scanned 1, 2, and 3 weeks after injection. In all mice a clear BLI signal, reported as the signal intensity, was visible in the lungs after 1 week (Figures 1a and 3), indicating the presence of metastasis to the lungs. Moreover, a significant longitudinal
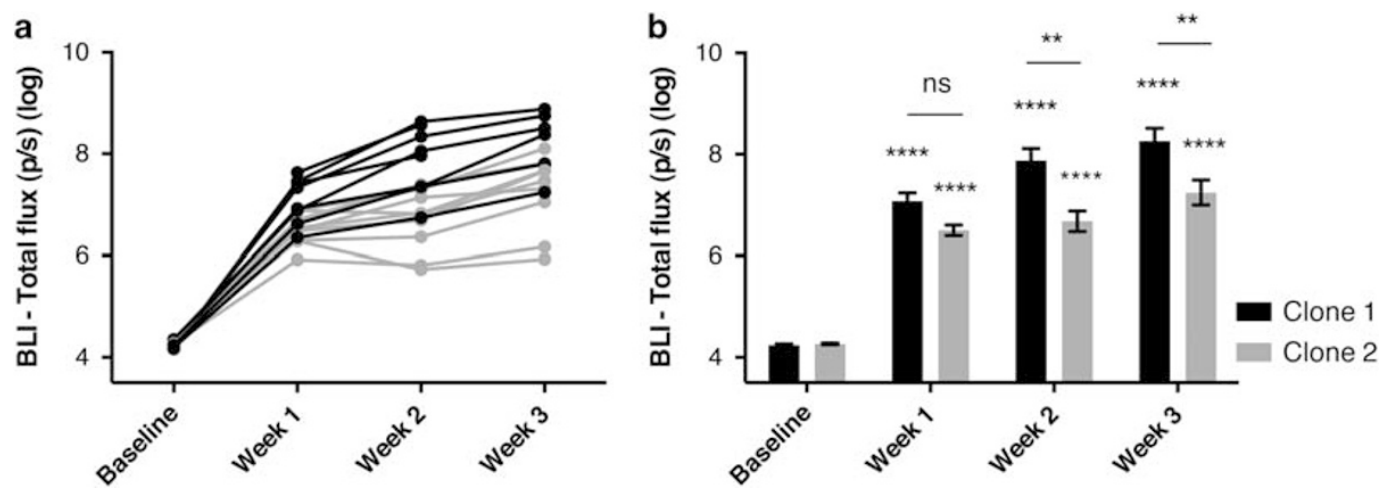

Figure 1 Longitudinal in vivo bioluminescence imaging (BLI) to monitor lung metastasis. Two individual clones of the lung squamous cell carcinoma (SCC) cell line KLN205 were intravenously injected in syngeneic DBA/2 mice and lung metastasis was monitored using BLI once a week during 3 weeks after injection. Graphs show longitudinal BLI signal intensity expressed as total flux (p/s). Graph (a) displays results for all mice individually ( $n=17$ (15 at week 3)) and graph (b) presents group results for mice injected with KLN205 cell clone 1 ( $n=8$ (6 at week 3)) and clone $2(n=9)$. Data represent mean \pm s.e.m. ${ }^{* *} P<0.01 ;{ }^{* * *} P<0.0001$. NS, not significant. 
increase of BLI signal intensity was observed for both clones (Figures $1 \mathrm{a}$ and $\mathrm{b}$ ), indicating an increase in tumor mass. Also, significant subject matching of longitudinal measurements was detected, which demonstrates the importance of longitudinal measurements, in which each animal serves as its own control, thereby taking into account the variability between animals.

Additionally, the difference in lung metastasis between both clones was evaluated. Significantly higher BLI signal intensities were detected for clone 1 compared with clone 2 after 2 and 3 weeks (Figure 1b). To eliminate the possibility that the observed differences in growth characteristics for both clones in vivo could be caused by intrinsic differences of both cell clones in vitro, in vitro proliferation and BLI signal intensities were assessed (Supplementary Figures S1A and B). All cell lines showed a similar growth pattern in the exponential phase (Supplementary Figure S1A) and similar BLI signal intensities (Supplementary Figure S1B). Therefore, the significantly higher in vivo BLI signal intensities observed for clone 1 compared with clone 2 can be attributed to higher levels of metastasis for this clone.

\section{Quantification of $\mu$ CT-Derived Lung Biomarkers Reveals Longitudinal Changes in Mice with Lung Metastasis}

To visualize both tumor and lung morphology in more detail, lung metastasis was evaluated in parallel using $\mu \mathrm{CT}$ scans of the same mice, acquired at baseline and weekly after injection of the two KLN205 cancer cell clones. Visual inspection of the $\mu \mathrm{CT}$ images showed that lung metastasis displayed highly diffuse tumor morphology, spread throughout the lung (Figure 3). Because of this diffuse morphology, metastasis was difficult to assess via visual inspection alone. To further evaluate how $\mu \mathrm{CT}$ scans can be used to detect and quantify lung metastasis, we quantified four lung biomarkers derived from the $\mu \mathrm{CT}$ scans that measure changes in different aspects of the lung because of the present pathology. We observed a global longitudinal increase in the lung tissue (including tumor) volume (Figures $2 \mathrm{a}$ and $\mathrm{b}$ ), which was significant compared with baseline after 2 and 3 weeks for clone 1 and clone 2 , respectively (Figure $2 \mathrm{~b}$ ). In contrast, the aerated lung volume showed a longitudinal decrease (Figures $2 \mathrm{c}$ and $\mathrm{d}$ ), resulting in significantly decreased air spaces at 3 weeks compared with baseline for both clones (Figure 2d). Remarkably, the size of the lungs as a whole, quantified by the total lung volume biomarker, gradually increased with time (Figures 2e and $\mathrm{f}$ ), with this increase in the total lung volume being already significant after 1 and 2 weeks for clone 1 and clone 2 , respectively (Figure $2 \mathrm{f}$ ). The mean lung density, a marker for the bulk of changes in the lungs (Figures $2 \mathrm{~g}$ and $\mathrm{h}$ ), followed the same evolution as the increase in lung tissue volume, reflecting the overall increase in lung density with time after metastasis induction. Also, for all the four $\mu \mathrm{CT}$ biomarkers, a significant matching of longitudinal measurements was observed, again strengthening the importance of longitudinal measurements within the same individual, as explained in the previous paragraph.

Subsequently, the four $\mu \mathrm{CT}$ biomarkers were evaluated with regard to their potential to detect differences in lung metastasis between two clones. Both the lung tissue volume (Figure 2b) and total lung volume (Figure 2f) were significantly higher for clone 1 compared with clone 2 at 3 weeks. The mean lung density (Figure $2 \mathrm{~h}$ ) followed the same trend, although the difference between both clones was not significant. As expected, the aerated lung volume followed the opposite trend, revealing a lower aerated lung volume for clone 1 compared with clone 2 (Figure $2 \mathrm{~d}$ ). The lower volume or air spaces in the lung for clone 1 is consistent with the higher lung tissue and tumor volume derived from the longitudinal $\mu \mathrm{CT}$ data for this clone.

As $\mu \mathrm{CT}$ imaging requires irradiation, a possible concern, however, may be that repeated $\mu \mathrm{CT}$ scanning may affect lung and tumor biology. Therefore, we compared mice that were weekly scanned via $\mu \mathrm{CT}$ with mice that were not. To permit a longer time course of tumor growth, we initially have injected less tumor cells. As shown in Supplementary Figures S2A and $\mathrm{B}$, no significant changes were observed 4 weeks after injection for both BLI and $\mu \mathrm{CT}$-derived biomarkers, confirming that the used irradiation dose did not affect lung metastasis in our model.

\section{Gold Standard Ex Vivo Readouts Confirm BLI and $\mu \mathrm{CT}$ Readouts for Lung Metastasis}

To validate noninvasive longitudinal BLI and $\mu \mathrm{CT}$ readouts against gold standard evaluation of lung metastasis, lungs were dissected after the last imaging time point at 3 weeks and prepared for histology. In Figure 3, representative examples are shown for mice with low, medium and high lung metastasis and their corresponding $\mu \mathrm{CT}$, BLI, and histology readouts. Clear correlations are visible between $\mu \mathrm{CT}$, BLI, and histology images. Moreover, the parallel increase in total lung volume, as quantified via $\mu \mathrm{CT}$, with increasing lung metastasis was confirmed in dissected lungs: lungs with advanced metastasis were clearly enlarged. Also, the decrease in aerated lung volume in combination with an increase in lung tissue volume that corresponds to higher lung metastasis based on $\mu \mathrm{CT}$ scans, could be observed in the corresponding lung tissue sections: less air spaces and increased tumor mass were obviously present in tissue sections of lungs with expanding metastasis. In conclusion, both imaging modalities were validated using ex vivo readouts.

\section{$\mu C T-D e r i v e d$ Measures Correlate with BLI and Describe Complementary Aspects of Lung Metastasis}

Based on our data, $\mu \mathrm{CT}$-derived biomarkers quantify changes in different aspects of the lung because of the present lung metastasis. To evaluate if those $\mu \mathrm{CT}$-derived measures correlate with BLI signal intensity, we studied the agreement between those biomarkers throughout the time course of metastasis. Significant correlations between each of the four 
different $\mu \mathrm{CT}$ biomarkers and BLI signal intensity were observed (Figure 4). For the lung tissue volume, the total lung volume and the mean lung density, a positive correlation was detected. For the aerated lung volume, as expected, a negative correlation was discovered, which is in line with the invasion of air spaces by tumor cells. For most of the $\mu \mathrm{CT}$ biomarkers, mentioned correlation with the BLI signal intensity was already present and significant at week 1, but is becoming
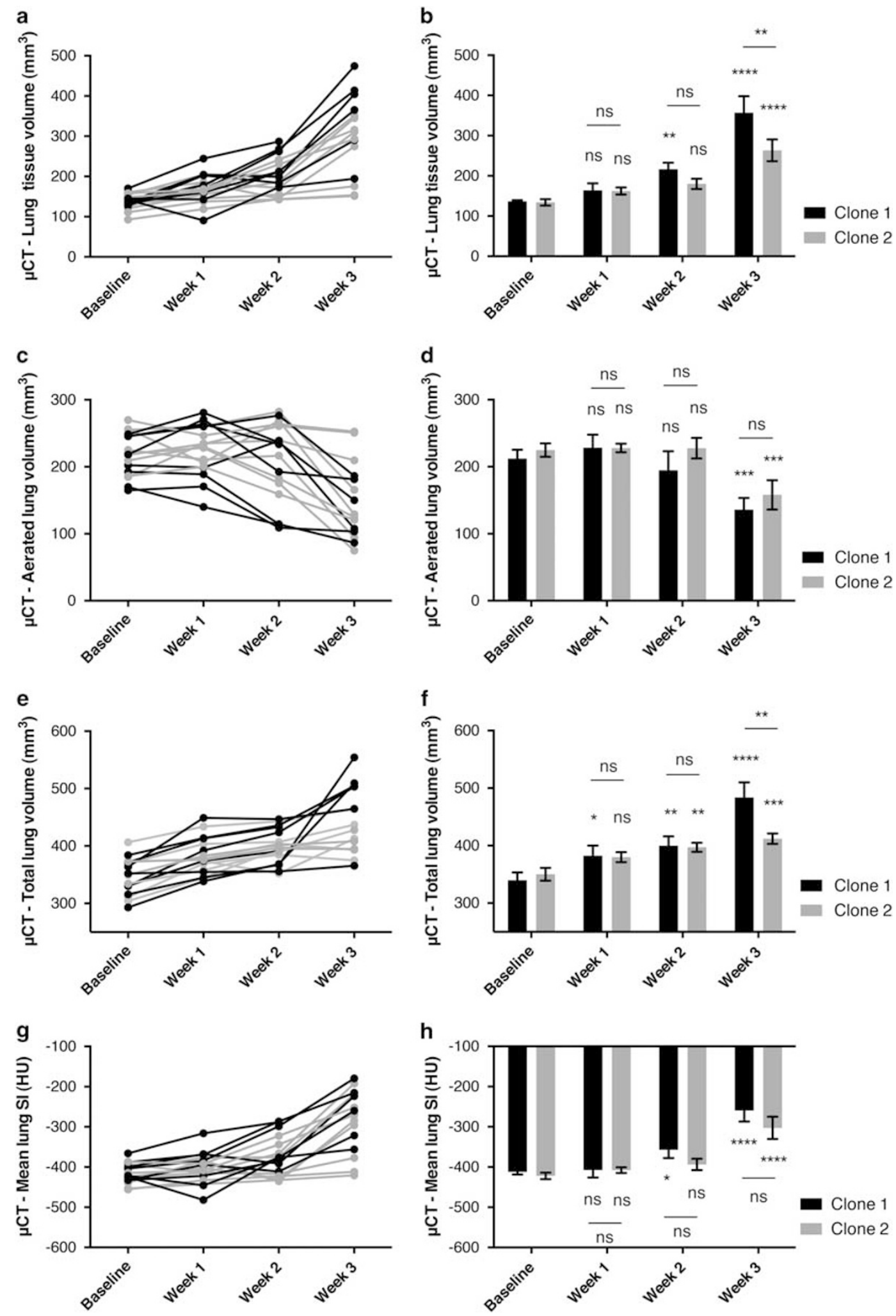
stronger towards week 3. The correlation between the lung tissue volume and BLI signal intensity was the strongest and most significant. The link with the total lung volume, however, was only present and significant at week 3 .

\section{DISCUSSION}

As novel research tools that allow longitudinal measurements of metastasis to the lungs in individual animals are highly needed in the cancer research field, we have monitored lung metastasis using $\mu \mathrm{CT}$ in addition to BLI and gold standard ex vivo readouts in a preclinical syngeneic mouse model. We have investigated in depth how both imaging modalities provide longitudinal information, from which several biomarkers can be quantified that describe different aspects of lung metastasis.

After intravenous injection of the cancer cell line KLN205 in syngeneic DBA/2 mice, a clearly visible BLI signal in all cases confirmed metastasis to the lungs 1 week after injection of the cancer cells. Moreover, a longitudinal increase of BLI signal intensity was observed for most mice, indicating an increase in tumor mass. However, its poor spatial resolution, semiquantitative nature due to surface weighting of the signal, lack of anatomical information and the need to genetically alter cancer cell lines limit the usefulness of BLI and drive the search towards novel tools that are capable of providing information on tumor morphology and lung anatomy.

In contrast and complementary to BLI, we demonstrate that $\mu \mathrm{CT}$ visualizes both tumor and lung morphology in more detail. We detected that lung metastasis in our model was very diffuse and spread throughout the lungs in contrast to clearly delineated lung tumors in previous reports. ${ }^{19-22}$ We therefore delineated the complete lungs to quantify the volume of hyperintense and hypointense signal via overall thresholding within the lung to quantify tumor mass and aerated lung volume, respectively. The aerated lung volume quantifies the air content of the lungs and probably best corresponds to the vital lung function. Additionally, we calculated the mean density for all voxels in the lungs and the overall lung volume. As such, we could extract four meaningful biomarkers from every $\mu \mathrm{CT}$ data set.

First of all, we observed a longitudinal increase in the lung tissue volume biomarker. Because it was previously shown that lung tissue volume remains stable in healthy mice, ${ }^{12}$ the observed increase of lung tissue volume in our study can be completely assigned to the increase in lung tumor mass. In line herewith is that the strongest correlation was found for this $\mu \mathrm{CT}$-derived biomarker and BLI signal intensity, as they both directly reflect tumor abundance.

Moreover, a longitudinal increase with increasing lung metastasis could also be detected for the total lung volume biomarker. The marked increase in total lung volume ( $28 \%$ increase at week 3 compared with $5 \%$ increase in healthy mice ${ }^{12}$ ) reflects the expansion of the lungs in response to tumor growth. This increase in total lung volume has also been observed in other lung diseases, such as lung fibrosis and infection. ${ }^{12}$

The aerated lung volume biomarker, on the other hand, appeared to decrease when lung metastasis was progressing, which corresponds to invasion of air spaces during metastasis. A similar decrease in aerated spaces was observed previously for lung fibrosis and infection, in which air spaces were invaded by fibrotic tissue and fungal lesions, respectively. ${ }^{12}$ However, the detected decrease in aerated lung volume in our model would be expected to be larger based on the extent of increase in lung tissue volume that was measured, which suggests that the increase in total lung volume affects the interpretation of the other biomarkers. A similar reasoning can apply to the mean lung density biomarker, as this marker represents the bulk of all changes in air and tissue (including tumor) content of the entire lung. Based on these observations, it is tempting to hypothesize that the increase in total lung volume would be a compensatory host response to the increase in tumor burden of the lungs, in an attempt to limit the loss of aerated lung spaces and hence vital lung function. It would be interesting to investigate whether this would be an active or passive mechanism. Nevertheless, $\mu \mathrm{CT}$ proves to be an indispensable tool to evaluate all changes in the lung during metastasis in preclinical models.

In addition, we aimed to evaluate the ability to assess possible differences in metastatic capacity between independent cellular clones using BLI and $\mu \mathrm{CT}$. Therefore, two individual KLN205 lung cancer cell clones were injected in $\mathrm{DBA} / 2$ mice. Both BLI and $\mu \mathrm{CT}$-derived measures enabled us to reveal differences in lung metastatic potential between the two clones. Higher metastasis was found for clone 1 compared with clone 2 , which was demonstrated by a higher BLI signal intensity, lung tissue volume, total lung volume and mean lung density, and a lower aerated lung volume for clone 1 compared with clone 2. Both BLI and $\mu \mathrm{CT}$ can therefore be used to assess sensitively differences between cell lines or conditions. As both clones in our study originated from the same cell line with similar in vitro growth pattern but behaved differently regarding lung metastasis, our results

Figure 2 Longitudinal in vivo lung microcomputed tomography $(\mu \mathrm{CT})$-derived biomarkers to monitor lung metastasis. Two individual clones of the lung squamous cell carcinoma (SCC) cell line KLN205 were intravenously injected in syngeneic DBA/2 mice and lung metastasis was evaluated using $\mu$ CT at baseline and once a week during 3 weeks after injection. Graphs present longitudinal changes of four lung biomarkers quantified from the $\mu \mathrm{CT}$ images. Graphs (a, c, e, and $\mathbf{g}$ ) show results for all individual mice ( $n=17$ (15 at week 3)) and graphs (b, d, $\mathbf{f}$, and $\mathbf{h})$ for groups of mice injected with KLN205 cell clone $1(n=8$ (6 at week 3)) and clone $2(n=9)$ for the following biomarkers: (a and b) lung tissue volume, (c and $\mathbf{d})$ aerated lung volume, (e and $\mathbf{f})$ total lung volume, and (g and $\mathbf{h})$ mean lung density. Data represent mean \pm s.e.m. ${ }^{*} P<0.05,{ }^{* *} P<0.01,{ }^{* *} P<0.001$, and ${ }^{* * * *} P<0.0001$. HU, Hounsfield Unit; NS, not significant. 

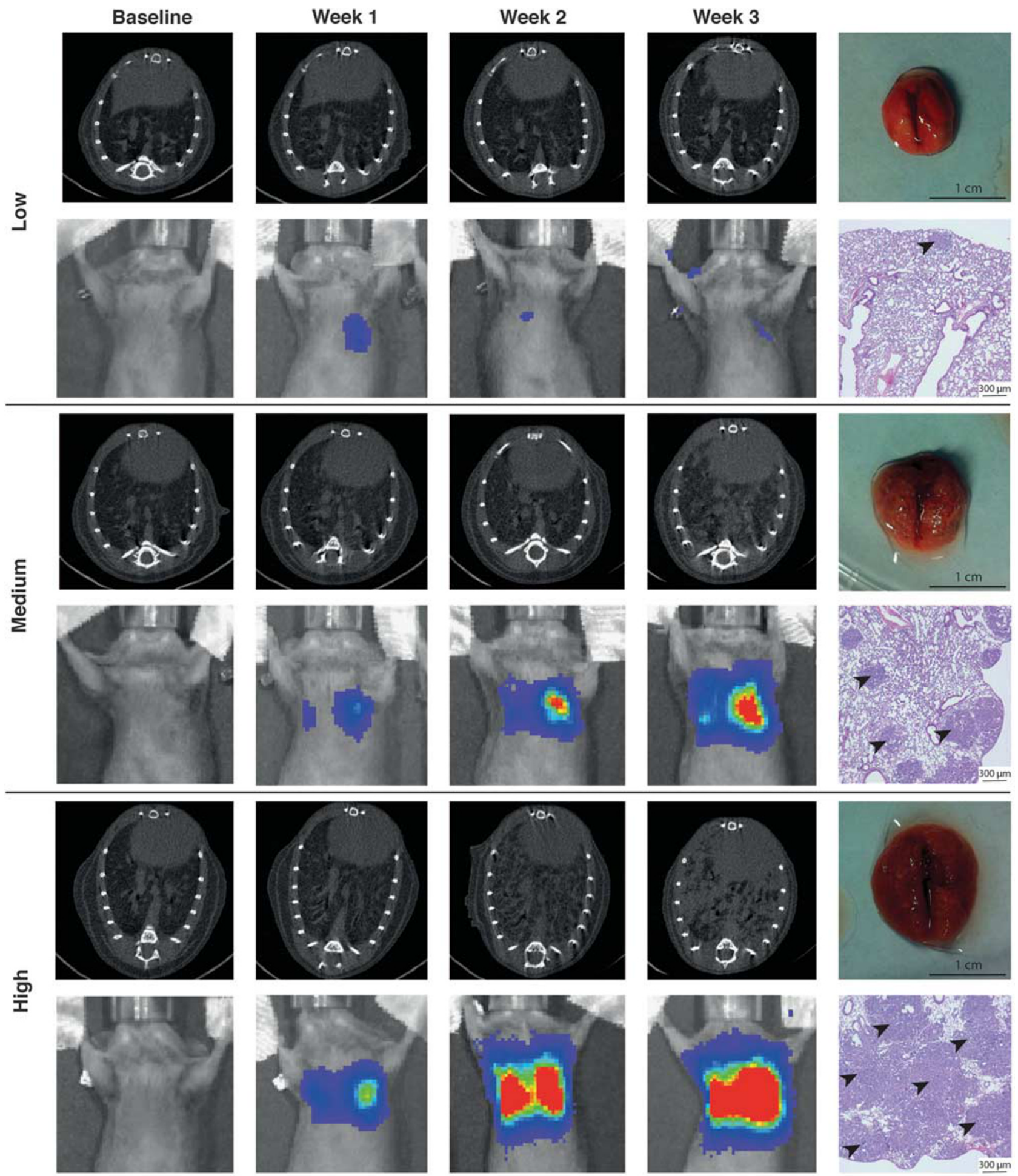

$$
\begin{array}{lccc}
1 \times 10^{6} & 2 \times 10^{6} \quad 3 \times 10^{6} & 4 \times 10^{6} & 5 \times 10^{6} \\
& \text { Radiance }\left(\mathrm{p} / \mathrm{s} / \mathrm{cm}^{2} / \mathrm{sr}\right)
\end{array}
$$

Figure 3 Representative bioluminescence imaging (BLI), microcomputed tomography ( $\mu \mathrm{CT}$ ), and ex vivo readouts. Lung BLI and $\mu \mathrm{CT}$ images at baseline and 1, 2, and 3 weeks after intravenous injection of KLN205 cells for a representative mouse with low, medium, and high lung metastasis load. $\mu \mathrm{CT}$ cross-sections of approximate similar level are shown. A photograph and hematoxylin-eosin (H\&E) staining of the corresponding dissected lungs are presented. Lung metastases are indicated by arrows. Note that not all metastases are indicated, but some are designated as examples. 

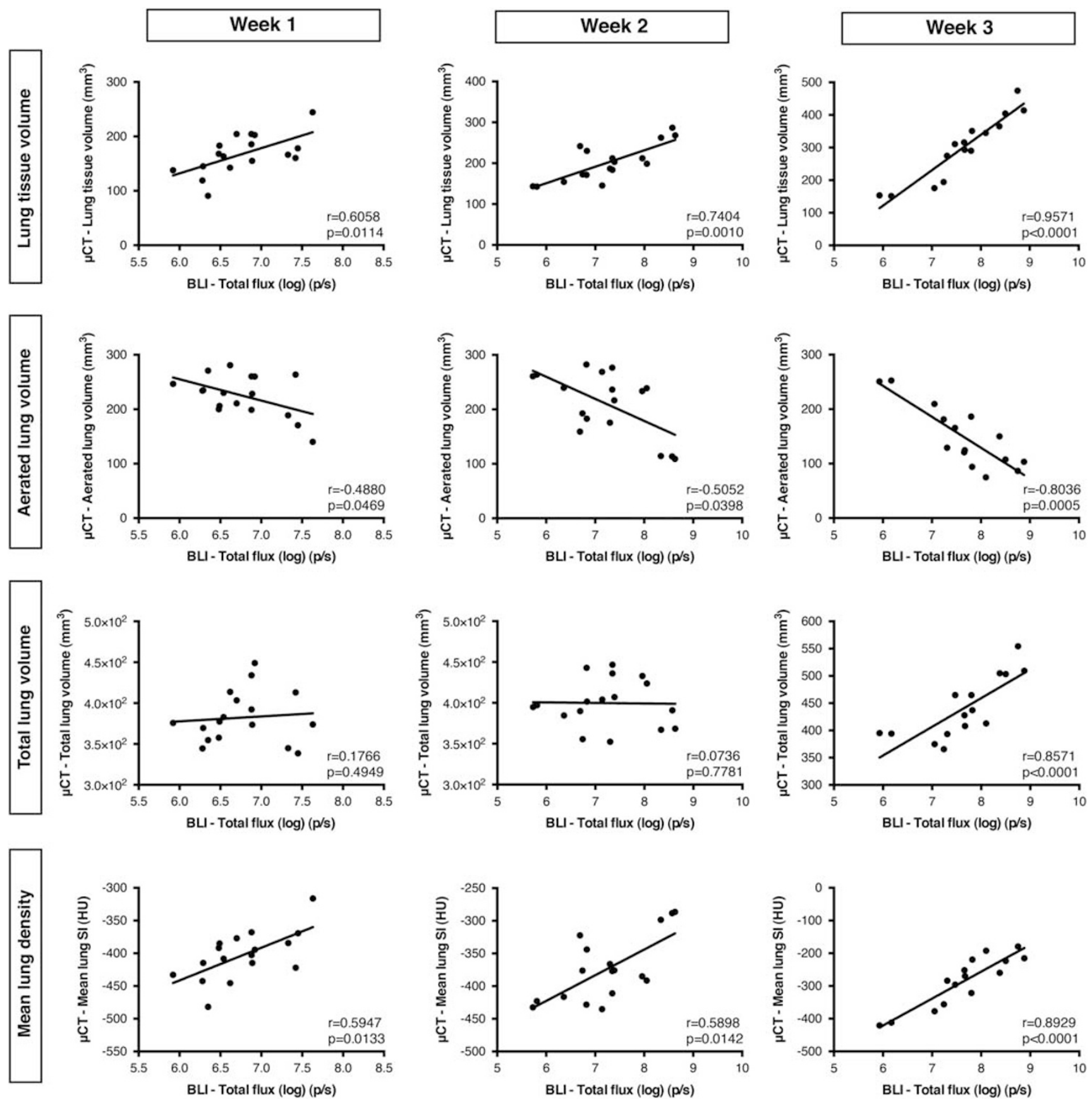

Figure 4 Correlation between quantitative microcomputed tomography $(\mu \mathrm{CT})$-derived biomarkers and bioluminescence imaging (BLI) signal intensity. Scatterplots for the assessment of correlation between the four different $\mu \mathrm{CT}$ parameters (lung tissue volume, aerated lung volume, total lung volume, and mean lung density) and total fluxes measured via BLI 1, 2 and 3 weeks after intravenous injection of both KLN205 cell clones ( $n=17$ (15 at week 3)). The Spearman's correlation coefficient and corresponding $P$-value are given on each graph. HU, Hounsfield Unit.

strengthen the importance to use at least two different independent clones as controls when single-cell clones need to be prepared.

To the best of our knowledge, this is the first study quantifying $\mu$ CT-derived biomarkers in a preclinical syngeneic model of lung metastasis, thereby addressing gaps in the field with regard to quantification of metastasis. Syngeneic mouse models recapitulate certain aspects of lung metastasis associated with the stroma, vascularity, and immune infiltrate more closely than human-mouse xenograft models, ${ }^{4-6}$ which are often used in previous reports. ${ }^{20,23,24} \mu \mathrm{CT}$-derived biomarkers are ideal for early detection of lung metastasis and for quantification of diffuse lung metastasis models in which the lung nodules are too small and plentiful to count, 
which is novel compared with previous reports where clearly delineated lung tumors or nodules were studied ${ }^{20-23}$ and where $\mu \mathrm{CT}$ only contributed anatomical information of the lungs. ${ }^{25}$ Moreover, quantification of live 3D imaging of metastasis is much more accurate, efficient, and useful compared with quantification of gold standard 2D ex vivo readouts at a single time point. An additional asset of $\mu \mathrm{CT}$ compared with BLI is that no genetic alteration and subsequent validation of cancer cell lines is needed. Although we did not find any evidence for altered growth of fluctransduced cells in vitro, this is still no watertight guarantee that genetically altered cells would not behave differently in vivo. This is an inherent potential limitation of BLI that requires transgenesis of cells. $\mu \mathrm{CT}$ offers an alternative for which no transgenesis of cells is required, obviating any residual risk of altering the biological properties of the cancer cell line under evaluation.

A possible concern may be that $\mu \mathrm{CT}$ imaging requires irradiation and may affect lung and tumor biology. However, we showed that repeated $\mu \mathrm{CT}$ scanning did not affect lung metastasis in our model. Moreover, in line with our results, radiation dose and dose rate of a single lung $\mu \mathrm{CT}$ examination is not expected to result in any tissue, cell, or DNA damage that could affect experimental outcome. ${ }^{26-30}$ Even a cumulative dose of several Grays during longitudinal $\mu \mathrm{CT}$ acquisitions should not be a concern, because animals are well able to recover from fractionated amounts and low dose rates of radiation exposure. ${ }^{30-35}$ Repeated $\mu \mathrm{CT}$ scanning using our high-resolution 4D protocol for 5 weeks and even up to 12 consecutive weeks did not induce any lung damage in healthy mice. ${ }^{36}$ Because the dose rate and dose delivered during our $\mu \mathrm{CT}$ study are both at least an order of magnitude smaller than the dose and rate applied in radiotherapy research, ${ }^{26-28,37-40}$ it is unlikely that metastases would be affected. This is supported by the lack of differences in Lewis lung carcinoma tumor volume found 21 days after irradiation with a therapeutic dose of $12 \mathrm{~Gy}$ compared with unirradiated tumor-bearing mice. ${ }^{41}$ Moreover, 4 weekly repeated $\mu \mathrm{CT}$ did not significantly alter the total tumor burden or the number of metastatic sites by comparing BLI results for metastatic human breast cancer between scanned and non-scanned mouse cohorts. ${ }^{42}$

In conclusion, in the present study we have monitored lung metastasis in a preclinical syngeneic mouse model using noninvasive BLI and $\mu \mathrm{CT}$-derived measures in real time. Both imaging modalities were crossvalidated via gold standard ex vivo readouts and offer biomarkers that describe different and complementary aspects of lung metastasis, underlining the importance of adding $\mu \mathrm{CT}$ to a multimodality follow-up. These findings are important to better assess lung metastasis in preclinical studies, which will be beneficial for translational applications.

Supplementary Information accompanies the paper on the Laboratory Investigation website (http://www.laboratoryinvestigation.org)

\section{ACKNOWLEDGMENTS}

Viral vectors were constructed and produced in the Leuven Viral Vector Core (LWC), and imaging was performed in the Molecular Small Animal Imaging Center (MoSAIC), both core facilities of the KU Leuven. Paraffin embedding and histology was carried out by the Translational Cell and Tissue Research facility (KU Leuven). We thank Tinne Buelens and Ann Van Santvoort for their excellent technical support. This research was supported by Grant 1506114N from the Research Foundation-Flanders (FWO) and CREA/14/015 from the KU Leuven (to GW) and Grant F/2014/324 from the STK Foundation against Cancer (to JVS). GW received a postdoctoral fellowship of the FWO. EM was supported by a fellowship from the Flemish League against Cancer (to VLK).

\section{DISCLOSURE/CONFLICT OF INTEREST}

The authors declare no conflict of interest.

1. ACS. Cancer Facts and Figures 2015. American Cancer Society: Atlanta, GA, USA, 2015

2. Hanahan D, Weinberg RA. The hallmarks of cancer. Cell 2000;100: 57-70.

3. Hanahan D, Weinberg RA. Hallmarks of cancer: the next generation. Cell 2011;144:646-674.

4. Khanna C, Hunter K. Modeling metastasis in vivo. Carcinogenesis 2005;26:513-523.

5. Krishnan K, Khanna C, Helman LJ. The molecular biology of pulmonary metastasis. Thorac Surg Clin 2006;16:115-124.

6. Junttila MR, de Sauvage FJ. Influence of tumour micro-environment heterogeneity on therapeutic response. Nature 2013;501:346-354.

7. Page DB, Postow MA, Callahan MK, et al. Immune modulation in cancer with antibodies. Annu Rev Med 2014;65:185-202.

8. Smyth MJ, Ngiow SF, Ribas A, et al. Combination cancer immunotherapies tailored to the tumour microenvironment. Nat Rev Clin Oncol 2016;13:143-158.

9. Rehemtulla A, Stegman LD, Cardozo SJ, et al. Rapid and quantitative assessment of cancer treatment response using in vivo bioluminescence imaging. Neoplasia 2000;2:491-495.

10. Soling A, Rainov NG. Bioluminescence imaging in vivo-application to cancer research. Expert Opin Biol Ther 2003;3:1163-1172.

11. Zinn KR, Chaudhuri TR, Szafran AA, et al. Noninvasive bioluminescence imaging in small animals. ILAR J 2008;49:103-115.

12. Vande Velde G, Poelmans J, De Langhe E, et al. Longitudinal micro-CT provides biomarkers of lung disease that can be used to assess the effect of therapy in preclinical mouse models, and reveal compensatory changes in lung volume. Dis Model Mech 2016;9:91-98.

13. De Langhe E, Vande Velde G, Hostens J, et al. Quantification of lung fibrosis and emphysema in mice using automated micro-computed tomography. PLoS One 2012;7:e43123.

14. Vande Velde G, De Langhe E, Poelmans J, et al. Longitudinal in vivo microcomputed tomography of mouse lungs: no evidence for radiotoxicity. Am J Physiol 2015;309:L271-L279.

15. Vande Velde G, De Langhe E, Poelmans J, et al. Magnetic resonance imaging for noninvasive assessment of lung fibrosis onset and progression: cross-validation and comparison of different magnetic resonance imaging protocols with micro-computed tomography and histology in the bleomycin-induced mouse model. Invest Radiol 2014;49:691-698.

16. Bland JM, Altman DG. Measuring agreement in method comparison studies. Stat Methods Med Res 1999:8:135-160.

17. Kaneko T, LePage GA. Growth characteristics and drug responses of a murine lung carcinoma in vitro and in vivo. Cancer Res 1978;38: 2084-2090.

18. Saito A, Morishita N, Mitsuoka C, et al. Intravenous injection of irradiated tumor cell vaccine carrying oncolytic adenovirus suppressed the growth of multiple lung tumors in a mouse squamous cell carcinoma model. J Gene Med 2011;13:353-361.

19. Bianchi A, Dufort $S$, Fortin PY, et al. In vivo MRI for effective noninvasive detection and follow-up of an orthotopic mouse model of lung cancer. NMR Biomed 2014;27:971-979.

20. lochmann $S$, Lerondel $S$, Blechet $C$, et al. Monitoring of tumour progression using bioluminescence imaging and computed tomography scanning in a nude mouse orthotopic model of human small cell lung cancer. Lung Cancer 2012;77:70-76. 
21. Fushiki $\mathrm{H}$, Kanoh-Azuma $\mathrm{T}$, Katoh $\mathrm{M}$, et al. Quantification of mouse pulmonary cancer models by microcomputed tomography imaging. Cancer Sci 2009;100:1544-1549.

22. Barck KH, Bou-Reslan H, Rastogi $\mathrm{U}$, et al. Quantification of tumor burden in a genetically engineered mouse model of lung cancer by micro-ct and automated analysis. Transl Oncol 2015;8:126-135.

23. Li XF, Zanzonico $P$, Ling CC, et al. Visualization of experimental lung and bone metastases in live nude mice by X-ray micro-computed tomography. Technol Cancer Res Treat 2006;5:147-155.

24. Davison CA, Chapman SE, Sasser TA, et al. Multimodal optical, X-ray CT, and SPECT imaging of a mouse model of breast cancer lung metastasis. Curr Mol Med 2013;13:368-376.

25. Adiseshaiah PP, Patel NL, lleva LV, et al. Longitudinal imaging of cancer cell metastases in two preclinical models: a correlation of noninvasive imaging to histopathology. Int J Mol Imag 2014;2014:102702.

26. Granton PV, Dubois L, van Elmpt W, et al. A longitudinal evaluation of partial lung irradiation in mice by using a dedicated image-guided small animal irradiator. Int J Radiat Oncol Biol Phys 2014;90:696-704.

27. Plathow C, Li M, Gong P, et al. Computed tomography monitoring of radiation-induced lung fibrosis in mice. Invest Radiol 2004;39:600-609.

28. Saito S, Murase K. Detection and early phase assessment of radiationinduced lung injury in mice using micro-CT. PLoS One 2012;7:e45960.

29. Boone JM, Velazquez O, Cherry SR. Small-animal X-ray dose from micro-CT. Mol Imag 2004;3:149-158.

30. Hudson D, Kovalchuk I, Koturbash I, et al. Induction and persistence of radiation-induced DNA damage is more pronounced in young animals than in old animals. Aging 2011;3:609-620.

31. Mole RH. Quantitative observations on recovery from whole body irradiation in mice. II. Recovery during and after daily irradiation. Br J Radiol 1957;30:40-46.

32. Detombe SA, Dunmore-Buyze J, Petrov IE, et al. X-ray dose delivered during a longitudinal micro-CT study has no adverse effect on cardiac and pulmonary tissue in C57BL/6 mice. Acta Radiol 2013;54:435-441.
33. Ford $\mathrm{NL}$, Martin $\mathrm{EL}$, Lewis JF, et al. In vivo characterization of lung morphology and function in anesthetized free-breathing mice using micro-computed tomography. J Appl Physiol 2007;102:2046-2055.

34. Steel GG, Down JD, Peacock JH, et al. Dose-rate effects and the repair of radiation damage. Radiother Oncol 1986;5:321-331.

35. Down JD, Easton DF, Steel GG. Repair in the mouse lung during low dose-rate irradiation. Radiother Oncol 1986;6:29-42.

36. Vande Velde $G$, De Langhe $E$, Poelmans J, et al. Longitudinal in vivo microcomputed tomography of mouse lungs: No evidence for radiotoxicity. Am J Physiol Lung Cell Mol Physiol 2015;309: L271-L279.

37. Rube $C E$, Uthe $\mathrm{D}$, Schmid $\mathrm{KW}$, et al. Dose-dependent induction of transforming growth factor beta (TGF-beta) in the lung tissue of fibrosis-prone mice after thoracic irradiation. Int J Radiat Oncol Biol Phys 2000;47:1033-1042.

38. Iwakawa M, Noda S, Ohta T, et al. Strain dependent differences in a histological study of CD44 and collagen fibers with an expression analysis of inflammatory response-related genes in irradiated murine lung. J Radiat Res 2004:45:423-433.

39. Bokobza SM, Jiang Y, Weber AM, et al. Short-course treatment with gefitinib enhances curative potential of radiation therapy in a mouse model of human non-small cell lung cancer. Int J Radiat Oncol Biol Phys 2014;88:947-954.

40. Gong H-Y, Hu W-G, Hu Q-Y, et al. Radiation-induced pulmonary injury accelerated pulmonary metastasis in a mouse model of breast cancer. Oncol Lett 2015;10:3613-3618.

41. Chen J, Wang $Y$, Mei Z, et al. Radiation-induced lung fibrosis in a tumor-bearing mouse model is associated with enhanced Type-2 immunity. J Radiat Res 2015;57:133-141.

42. Adams A, Cowey S, Kappes J, et al. Bioluminescence imaging confirms that weekly computed tomography studies do not change tumor growth in an animal model of breast cancer metastasis. Mol Imag Biol 2006:8:76-77. 